impossibility of $+T$ effects. The nitroso-group exhibits probably the strongest $-T$ effect yet recorded, but differs from nitroxyl, which can activate the halogen in para-halogenonitrobenzenes by virtue of the $-T$ mechanism (alkaline reagents), but which directs an entrant nitro-group into the meta position of nitrobenzene by reason of the permanent $-I$ effect, in that, by not possessing an ionic positive charge, $+T$ effects with the appropriate reagent will occur much more readily. Para substitutions take place by valency redistributions of this type occasioned by cationoid reagents; thus the direct para bromination and nitration of nitrosobenzene on one hand and the labilisation of bromine in para-bromonitrosobenzene on the other can be easily understood. The nitrosogroup is unique but not anomalous.

A correct formula for this substance must, however, indicate more than (1) and (2) together show. Nitrosobenzene, like other Ar-nitroso compounds, possesses properties reminiscent of an odd electron moleculefor example, in some compounds the - NO group is apparently paramagnetic - and qualitatively resembles a free diarylamino-ion ${ }^{8}$ in several respects. It thus is a case where ordinary chemical formulæ become inadequate.

A formulation involving a 3-electron bond has been implicitly stated by Pauling ${ }^{9}$ which is satisfactory for unexcited nitrosobenzene (intermediate between (1) and (2)), which thus becomes (3) - which will, like the blue free diarylamino-radical, not only polymerise readily to a colourless dimeride (4) containing tercovalent nitrogen, but also will in different

$$
\begin{array}{rrl}
\mathrm{Ph}: \mathrm{N}: \mathrm{O}: & \mathrm{Ph}: & \ddot{\mathrm{N}}: \ddot{\mathrm{O}}: \\
\cdots & : \ddot{\mathrm{O}}: \ddot{\mathrm{N}}: \mathrm{Ph} \\
& & . \cdot
\end{array}
$$

(3)

(4)

reactions exhibit opposite polarisations according to the requirements of the reagent. Incidentally, on these formulæ, both mono- and di-meric forms should produce para-substitution products, although the latter more easily than the former.

Pauling's arguments applied mutatis mutandis to the arsinoso-radical show that arsenic should be normally tercovalent, and that therefore no special analogy with the nitroso-group can be expected. An examination of 4-arsinosodimethylaniline has confirmed this.

R. J. W. LE Fìve.

The Ralph Forster Laboratories of

Organic Chemistry,

University College,

London, W.C.I.

1 Unpublished experiments.

2 Bamberger, Büsdorf, and Sand, Ber, 31, 15T3 1898.

Ingold, J.C.S., 516; 1925 : and Bamberger, Ber., 30, 512 ; 1897. 4 Ingold, loc. cit.

5 Ham, Dissert., Zurich, 29; 1904.

- Le Fèvre, J.C.S., 810; 1931 .

7 Hammick and Iilingworth, J.C.S., $2363 ; 1930$

- Wieland, "Die Hydrazine", Stuttgart, $71 ; 1913$.

- Pauling, J. Amer. Chem. Soc., 53, 3234; 1931.

\section{Constitution of the Keratin Molecule}

Is dealing with my ${ }^{1}$ comments upon their communication ${ }^{2}$ under this title, Speakman and Hirst ${ }^{3}$ describe as erroneous my statement that "the guanidine group of arginine does not ... yield nitrogen with nitrous acid under the usual conditions". By " usual conditions" I naturally imply those generally adopted in determinations of aminonitrogen in the course of protein analysis as described by van Slyke, ${ }^{4} 5$ that is to say, contact between acid nitrate solution and amino-body at room temperature, for 3-5 minutes in the case of mono-amino acids and for $\frac{1}{2}-1$ hour in the case of the hexone bases or proteins. Under these conditions the guanidine group of arginine is practically unaffected. The work of Plimmer ${ }^{6}$ to which Speakman and Hirst refer is, of course, well known to me, and in that paper it is stated that any error introduced by reaction of the guanidine group of arginine in a 1-hour determination is inappreciable. With longer reaction times, correspondingly greater decomposition does occur.

In connexion with my second point concerning the importance of taking into account the amide nitrogen of wool in the calculations originally put forward by them, Speakman and Hirst have restated their case by assuming that all the arginine and lysine in wool is combined with glutamic and aspartic acids, and demonstrating that an excess of these acids would still be available to combine with $0 \cdot 4$ per cent of amide nitrogen.

The total amide nitrogen of wool, as determined by Marston, ${ }^{7}$ is 1.2 per cent. No less than 0.8 per cent is thus left unaccounted for.

Speakman and Hirst consider that Abderhalden's figures for the proportion of dicarboxylic acids is low, but such a discrepancy would require that, in terms of glutamic acid, the figure at present accepted (12.9 per cent) is inaccurate by no less than 40 per cent in terms of weight, by 8.4 grams per 100 grams of wool. As I pointed out in my previous comments, Abderhalden and Voitinovici's 8 analysis of wool is the only complete one which is available, and I am glad to see that fresh determinations of aspartic and glutamic acids are being made in the University Laboratories at Leeds. Reliable data upon the composition of wool are sadly needed. Until these results are available, it is scarcely profitable to indulge in further speculation, although, considering the relative case of the determination of the two dicarboxylic acids, either separately or together, I am surprised at the confidence with which Speakman and Hirst prophesy that errors of such magnitude as 40 per cent will be found in the figures we already possess.

Claude Rimington.

Onderstepoort Veterinary Research Laboratory, Pretoria, South Africa, July 13.

1 Rimington, NATURE, 129, 580, April 16, 1932.

Speakman and Hirst, NATURE, 128, 1073, Dec. 26, 1931

Speakman and Hirst, NATURE, 129, 938, June 25, 1932

- Van Slyke, J. Biol. Chem., 9, 185 ; 1911.

- Vin Slyke, $J$. Biol. Chem., 10, 15;1911-12.

- Plímmer, Biochem. J., 18, 105 ; 1924.

? Marston, Council of Sei. and Ind. Res., Commonwealth of Australia, Bulletin 38; 1928.

8 Abderhalden and Voitinovici, Z. Physiol. Chem., 52, 368; 1907.

Dr. Rimington has no new point to raise. Originally, he had two difficulties, one of which has apparently been eliminated by my reply to his earlier communication. As regards the second, his difficulty has been reduced to a doubt whether re-determination of the glutamic and aspartic acid content of wool will give a sufficient increase to account precisely for the amide nitrogen as well as arginine and lysine. Even this doubt must disappear in the light of Jones and Moeller's 1 determinations of aspartic and glutamic acids in various proteins. These authors state that "new determinations of these amino acids have been made in a number of typical proteins, and invariably higher results for aspartic acid were obtained than those previously obtained by the ester method. In several cases from 4 to 9 times as much was found."

Textile Chemistry Laboratory,

$$
\text { J. B. Speakman. }
$$

Aug. 12.

2 Jones and Moeller, J. Biol. Chem., 79, 429 ; 1928.

No. 3280 , VoL. 130] 